\title{
Existence and Boundedness of Solutions for Nonlinear Volterra Difference Equations in Banach Spaces
}

\author{
Rigoberto Medina \\ Departamento de Ciencias Exactas, Universidad de Los Lagos, Casilla 933, Osorno, Chile \\ Correspondence should be addressed to Rigoberto Medina; rmedina@ulagos.cl
}

Received 25 June 2016; Accepted 30 October 2016

Academic Editor: Jozef Banas

Copyright (C) 2016 Rigoberto Medina. This is an open access article distributed under the Creative Commons Attribution License, which permits unrestricted use, distribution, and reproduction in any medium, provided the original work is properly cited.

\begin{abstract}
We consider a class of nonlinear discrete-time Volterra equations in Banach spaces. Estimates for the norm of operator-valued functions and the resolvents of quasi-nilpotent operators are used to find sufficient conditions that all solutions of such equations are elements of an appropriate Banach space. These estimates give us explicit boundedness conditions. The boundedness of solutions to Volterra equations with infinite delay is also investigated.
\end{abstract}

\section{Introduction}

In many phenomena of the real world, not only does their evolution prove to be dependent on the present state, but it is essentially specified by the entire previous history. These processes are encountered in the theory of viscoelasticity $[1,2]$, optimal control problems [3], and also description of the motion of bodies with reference to hereditary [3-5]. The mathematical description of these processes can be carried out with the aid of equations with the aftereffect, integral, and integrodifferential equations. A significant contribution to the development of this direction was made by V. Volterra, V. B. Kolmanovskii, N. N. Krasovskii, S. M. V. Lunel, A. D. Myshkis, and J. K. Hale.

The aim of this article is to develop a technique for investigating stability and boundedness of nonlinear implicit Volterra difference systems described by Volterra operator equations. Only a few papers deal with the theory of general Volterra equations and most of them are devoted to the stability analysis of explicit Volterra linear difference equations with constant coefficients or of convolution type. For example, in Minh [6], some results on asymptotic stability and almost periodicity are stated in terms of spectral properties of the equations and their solutions, which are linear Volterra equations of convolution type. In Minh [7], the asymptotic behavior of individual orbits of linear functional operator equations are established using an extension of the Katznelson-Tzafriri's theorem [8]. In Nagabuchi [9], using the decomposition of the phase space, together with the variation of constants formula in the phase space, the existence of almost periodic solutions for forced linear Volterra difference equations in Banach spaces is derived. The considered equations are of convolution type. In Murakami and Nagabuchi [10], sufficient stability properties and the asymptotic almost periodicity for linear Volterra difference equations in Banach spaces are derived. González et al. [11] consider an implicit nonlinear Volterra difference equation in a Hilbert space and obtained sufficient conditions so that the solutions exist and have a bounded behavior. The coefficients of the considered equations are sequences of real numbers. In Song and Baker [12], several necessary and sufficient conditions for stability are obtained for solutions of the linear Volterra difference equations by considering the equations in various choices of Banach spaces. However, the main results of this article are established essentially to implicit linear Volterra difference equations. In Muroya and Ishiwata [13], considering a nonlinear Volterra difference equation with unbounded delay, sufficient conditions for the zero solution to be globally asymptotically stable are derived. However, only an explicit scalar Volterra difference is considered. In Győri and Horváth 
[14], sufficient conditions are presented under which the solutions to a linear nonconvolution Volterra difference equation converge to limits, which are given by a limit formula. In Kolmanovskii et al. [15], stability and boundedness problems of some class of scalar Volterra nonlinear difference equations are investigated. Stability conditions and boundedness are formulated in terms of the characteristics equations. In Song and Baker [16], the fixed point theory is used to establish sufficient conditions to ensure the stability of the zero solution of an implicit nonlinear Volterra difference equation. Besides, the existence of asymptotically periodic solutions is established. However, in this article equations with linear kernel are mainly considered.

One of the basic methods in the theory of stability and boundedness of Volterra difference equations is the direct Lyapunov method (see $[1,3,17,18]$ ). But finding the Lyapunov functionals for Volterra difference equations is still a difficult task.

In this paper, to establish boundedness conditions of solutions, we will interpret the Volterra difference equations with nonlinear kernels as operator equations in appropriate spaces. Such an approach for linear Volterra difference equations has been used by Myshkis [5], Kolmanovskii et al. [15], Kwapisz [19], and Medina [20, 21]. The knowledge of these bounds is important because they represent the error between the exact solution of the original problem and its difference approximation.

Existence and uniqueness problems for the Volterra difference equations were discussed by some authors. Usually the solutions were sought in the phase $\operatorname{space} l_{p}^{\omega}\left(Z^{+}, X\right), \omega>0$ and $Z^{+}=\{0,1,2, \ldots\}$ (e.g., see $\left.[15,19]\right)$. In this paper, formulating the Volterra discrete equations in the phase space $l_{p}\left(Z^{+}, X\right)$, where $X$ is an appropriate Hilbert space, and assuming that the kernel operator has the Volterra property, we obtain sufficient conditions for the existence and uniqueness problem.

Our results compare favorably with the above-mentioned works in the following sense:

(a) Sufficient conditions for the existence and uniqueness of solutions of implicit nonlinear Volterra difference equations are obtained.

(b) We established a theory on the asymptotic behavior of implicit nonlinear Volterra difference systems which are described by Volterra operator equations.

(c) Explicit estimates for the solutions of nonlinear Volterra operator equations in Hilbert spaces are derived.

The remainder of this article is organized as follows: in Section 2, we introduce some notations, preliminary results, and the statement of the problem. In Section 3, the boundedness of solutions is derived using norm-estimates for the resolvents of completely continuous quasi-nilpotent operators. In Section 4, we discuss the boundedness of solutions of infinite-delay Volterra difference equations. Finally, Section 5 is devoted to the discussion of our results: we highlight the main conclusions.

\section{Statement of the Problem}

Let $X$ be a complex Hilbert space with norm $\|\cdot\|_{X}:=\|\cdot\|$. Let $l_{p}=l_{p}\left(Z^{+}, X\right)$ and $l_{\infty}=l_{\infty}\left(Z^{+}, X\right)$ be the spaces of sequences $h=\{h(j)\}_{j=1}^{\infty}$ such that

$$
\begin{aligned}
& l_{p}=\left\{h: Z^{+} \longrightarrow X: \sum_{j=1}^{\infty}\|h(j)\|^{p}<\infty\right\}, \\
& l_{\infty}=\left\{h: Z^{+} \longrightarrow X: \sup _{j \in Z^{+}}\|h(j)\|<\infty\right\} .
\end{aligned}
$$$$
1 \leq p<\infty
$$

The spaces $l_{p}, 1 \leq p \leq \infty$, equipped with the standard norms

$$
\begin{aligned}
\|h\|_{p} & =\left(\sum_{j=1}^{\infty}\|h(j)\|^{p}\right)^{1 / p}, \text { for } 1 \leq p<\infty, \\
\|h\|_{\infty} & =\sup _{j \in \mathbb{Z}^{+}}\|h(j)\|,
\end{aligned}
$$

are Banach spaces.

Denote

$$
\Omega_{r}=\left\{h \in l_{\infty}:\|h\|_{\infty} \leq r\right\}, \quad \text { for } 0<r \leq \infty .
$$

We consider Volterra difference equations on a separable Hilbert space $X$

$$
x(n)=f(n)+\sum_{j=0}^{n} K(n, j, x(j)), \quad n \geq 0,
$$

where

$$
K: Z^{+} \times Z^{+} \times\left\{\xi \in X:\|\xi\|_{\infty}<r\right\} \rightarrow X
$$

is the kernel, $Z^{+}$denotes the set of nonnegative integers, and $f: Z^{+} \rightarrow X$ is a given sequence.

Equation (4) can be regarded as the discrete-time analog of the classical integral Volterra equation of the second kind

$$
x(t)=f(t)+\int_{0}^{t} K(t, s, x(s)) d s, \quad(t \geq 0) .
$$

We point out the distinction between (4) and the explicit Volterra equation

$$
x(n)=f(n)+\sum_{j=0}^{n-1} K(n, j, x(j)), \quad n \geq 0 .
$$

The first step is to establish the solvability of (4). In the linear case, in which we write (4) as

$$
x(n)=f(n)+\sum_{j=0}^{n} K(n, j) x(j), \quad n \geq 0,
$$

with $K(n, j)$ being a finite-dimensional matrix, the existence of $x(n)$ for each $n$ follows from the property that $(I-K(n, n))$ 
is invertible for each $n \geq 0$. Song and Baker [16] deal with the existence of solutions of (4), defined on $d$-dimensional Euclidean spaces. In fact, considering implicit equations of the form

$$
x(n+1)=\psi_{n}(x(0), x(1), \ldots, x(n), x(n+1))
$$

and assuming appropriate conditions on $\left\{\psi_{n}\right\}$, they conclude that solutions of equations

$$
x=\psi_{n}\left(u_{0}, u_{1}, \ldots, u_{n}, x\right)
$$

are unique and can be expressed in the form

$$
x=\varphi_{n}\left(u_{0}, u_{1}, \ldots, u_{n}\right) .
$$

We present a different and more general approach to the problem of existence and uniqueness discussed in [16]. Namely, for a given general discrete operator $T: S\left(Z^{+}, X\right) \rightarrow$ $S\left(Z^{+}, X\right)$, consider the equation

$$
u(n)=(T u)(n), \quad n \in Z^{+},
$$

where $S\left(Z^{+}, X\right)$ denotes the linear space of all sequences $s$ : $Z^{+} \rightarrow X$.

We ask when the solutions of this equation belong to the space $l_{p}\left(Z^{+}, X\right)$ of all functions $x \in S\left(Z^{+}, X\right)$ satisfying the condition

$$
\left(\sum_{j=1}^{\infty}\|x(j)\|^{p}\right)^{1 / p}<\infty, \quad \text { for } 1 \leq p \leq \infty .
$$

Assumption A. The operator $T$ is a causal operator; that is, for any $n \in Z^{+}$,

$$
\begin{aligned}
(T u)(n)=(T v) & (n), \\
& \text { if } u(j)=v(j) \text { for } j=0,1, \ldots, n-1 .
\end{aligned}
$$

That is, the value of $(T u)$ at $n$ is determined by the values of $u(j)$ for $j=0,1, \ldots, n-1$ (e.g., see Corduneanu [22]).

Remark 1. The causal property of the operator $T$ guarantees the existence and uniqueness of the solutions of (12). Consequently, when the operator $T$ of (12) has, for instance, the form

$$
(T x)(n)=f(n)+\sum_{j=0}^{n} K(n, j, x(j)),
$$

we can establish existence and uniqueness results for the solutions of (4).

Theorem 2. If the operator $T$ defined in (15) is a causal operator, then there exists a solution of (4).

Finally, we will determine sufficient conditions on the coefficients of (4) such that its solutions belong to the space $l_{p}\left(Z^{+}, X\right), 1 \leq p \leq \infty$.

In the finite-dimensional case, the spectrum of a linear operator consists of its eigenvalues. The spectral theory of bounded linear operators on infinite-dimensional spaces is an important but challenging area of research. For example, an operator may have a continuous spectrum in addition to, or instead of, a point spectrum of eigenvalues. A particularly simple and important case is that of compact, self-adjoint operators. Compact operators may be approximated by finitedimensional operators, and their spectral theory is close to that of finite-dimensional operators.

To formulate the next result, let us introduce the following notations and definitions: let $H$ be a separable Hilbert space and $A$ a linear compact operator in $H$. If $\left\{e_{k}\right\}_{k=1}^{\infty}$ is an orthogonal basis in $H$ and the series $\sum_{k=1}^{\infty}\left(A e_{k}, e_{k}\right)$ converges, then the sum of the series is called the trace of the operator $A$ and is denoted by

$$
\operatorname{trace}(A)=\operatorname{tr}(A)=\sum_{k=1}^{\infty}\left(A e_{k}, e_{k}\right) .
$$

Definition 3. An operator $A$ satisfying the relation $\operatorname{tr}\left(A^{*} A\right)<$ $\infty$ is said to be a Hilbert-Schmidt operator, where $A^{*}$ is the adjoint operator of $A$.

The norm

$$
N_{2}(A)=N(A)=\sqrt{\operatorname{tr}\left(A^{*} A\right)}
$$

is called the Hilbert-Schmidt norm of $A$.

Definition 4. A bounded linear operator $A$ is said to be quasiHermitian if its imaginary component

$$
A_{I}=\frac{A-A^{*}}{2 i}
$$

is a Hilbert-Schmidt operator, where $A^{*}$ is the adjoint operator of $A$.

Theorem 5 (see [23, Lemma 2.3.1]). Let $V$ be a HilbertSchmidt completely continuous quasi-nilpotent (Volterra) operator acting in a separable Hilbert space $H$. Then the inequality

$$
\left\|V^{k}\right\| \leq \frac{N^{k}(V)}{\sqrt{k !}}, \text { for any natural } k,
$$

is true.

\section{Main Results}

Let $A_{r}$ denote the infinite matrix with components

$$
A_{r, n, j}:=\sup _{z \in X:\|z\| \leq r} \frac{\|K(n, j, z)\|}{\|z\|} ; \text { for } n, j=1,2, \ldots
$$

Assume that

$$
N_{p}\left(A_{r}\right)=\left[\sum_{n=1}^{\infty}\left(\sum_{j=1}^{n} A_{r, n, j}^{q}\right)^{p / q}\right]^{1 / q}<\infty,
$$

which implies

$$
\beta_{p}\left(A_{r}\right)=\sup _{n=1,2, \ldots}\left[\sum_{j=1}^{n} A_{r, n, j}^{q}\right]^{1 / q}<\infty
$$

where $1 / p+1 / q=1$. 
Denote

$$
m_{p}\left(A_{r}\right)=\sum_{j=0}^{\infty} \frac{N_{p}^{j}\left(A_{r}\right)}{\sqrt[p]{j !}} .
$$

Theorem 6. Assume that $f \in l_{p}\left(Z^{+}, X\right)$ and condition (21) holds. Then any solution $x=\left(x_{1}, x_{2}, \ldots\right)$ of (4) belongs to $l_{p}\left(Z^{+}, X\right)$ and satisfies the inequalities

$$
\begin{aligned}
\|x\|_{p} & \leq m_{p}\left(A_{r}\right)\|f\|_{p}, \\
\|x\|_{\infty} & \leq\|f\|_{\infty}+\beta_{p}\left(A_{r}\right) m_{p}\left(A_{r}\right)\|f\|_{p},
\end{aligned}
$$

provided that

$$
\|f\|_{\infty}+\beta_{p}\left(A_{r}\right) m_{p}\left(A_{r}\right)\|f\|_{p}<r .
$$

Proof of Theorem 6. We will decompose the proof of Theorem 6 in the following lemmas.

Lemma 7. Assume that $f \in l_{p}\left(Z^{+}, X\right), 1 \leq p<\infty$, and condition (21) holds with $r=\infty$, that is, $A_{\infty}$ with components

$$
A_{\infty}(n, j)=\sup _{z \in X} \frac{\|K(n, j, z)\|}{\|z\|}, \quad n, j=1,2, \ldots,
$$

is a Hilbert-Schmidt kernel. Then, any solution $x=\left(x_{1}, x_{2}, \ldots\right)$ of (4) belongs to $l_{p}\left(Z^{+}, X\right)$ and satisfies the inequality

$$
\|x\|_{p} \leq m_{p}\left(A_{\infty}\right)\|f\|_{p} .
$$

Proof. From (4) we have

$$
\|x(n)\| \leq \sum_{j=1}^{n} A_{\infty}(n, j)\|x(j)\|+\|f(n)\| .
$$

Let $L$ be the linear operator defined on $l_{p}\left(Z^{+}, X\right)$ by

$$
(L h)(n)=\sum_{j=1}^{n} A_{\infty}(n, j) h(j), \quad h \in l_{p}\left(Z^{+}, X\right) .
$$

Rewrite equation (4) as

$$
x=f+L x .
$$

Hence,

$$
x=(I-L)^{-1} f \text {. }
$$

Since

$$
(I-L)^{-1}=\sum_{j=0}^{\infty} L^{j}
$$

we have

$$
\left\|(I-L)^{-1}\right\|_{p} \leq \sum_{j=0}^{\infty}\left\|L^{j}\right\|_{p}
$$

Thus, by (32),

$$
\|x\|_{p} \leq \sum_{j=0}^{\infty}\left\|L^{j}\right\|_{p}\|f\|_{p} .
$$

Since $L$ is a quasi-nilpotent Hilbert-Schmidt operator, it follows, by Gil' [23, Lemma 2.3.1], that

$$
\left\|L^{k}\right\|_{p} \leq \frac{N_{p}^{k}\left(A_{\infty}\right)}{\sqrt[p]{k !}} .
$$

This and (35) yield the required result.

Remark 8. By Hölder's inequality, we have

$$
m_{p}\left(A_{\infty}\right)=\left(1-\frac{1}{p^{q / p}}\right)^{-1 / q} \exp \left[N_{p}^{p}\left(A_{\infty}\right)\right] .
$$

Consequently, from Lemma 7,

$$
\|x\|_{p} \leq \gamma_{p} \exp \left[N_{p}^{p}\left(A_{\infty}\right)\right]\|f\|_{p},
$$

where

$$
\gamma_{p}=\left(1-\frac{1}{p^{q / p}}\right)^{-1 / q} .
$$

Lemma 9. Assume that $f \in l_{\infty}\left(Z^{+}, X\right)$ and condition (21) holds with $r=\infty$, and then any solution $x=\left(x_{1}, x_{2}, \ldots\right)$ of (4) belongs to $l_{\infty}\left(Z^{+}, X\right)$ and satisfies the inequality

$$
\|x\|_{\infty} \leq\|f\|_{\infty}+\beta_{p}\left(A_{\infty}\right) m_{p}\left(A_{\infty}\right)\|f\|_{p} .
$$

Proof. From (31), it follows that

$$
\|x\|_{\infty} \leq\|f\|_{\infty}+\|L x\|_{\infty} .
$$

But due to Hölder's inequality

$$
\begin{aligned}
\|L x\|_{\infty} & \leq \sup _{n=1,2, \ldots}\left[\sum_{k=1}^{n} A_{\infty}^{q}(n, k)\right]^{1 / q}\|x\|_{p} \\
& =\beta_{p}\left(A_{\infty}\right)\|x\|_{p} .
\end{aligned}
$$

Hence, (28) and (42) yield

$$
\|x\|_{\infty} \leq\|f\|_{\infty}+\beta_{p}\left(A_{\infty}\right) m_{p}\left(A_{\infty}\right)\|f\|_{p} .
$$

Now, we will complete the Proof of Theorem 6, considering the case $0<r<\infty$.

By Urysohn's Lemma [24, p. 15], there are scalar-valued functions $\lambda_{r}$ and $\mu_{r}$ defined on $l_{p}\left(Z^{+}, X\right)$ and $X$, respectively, such that

$$
\lambda_{r}(h)= \begin{cases}1, & \|h\|_{\infty} \leq r \\ 0, & \|h\|_{\infty}>r\end{cases}
$$$$
\mu_{r}(h)= \begin{cases}1, & \|h\| \leq r, \\ 0, & \|h\|>r .\end{cases}
$$ 
Define

$$
\begin{aligned}
K_{r}(n, j, z) & =\lambda_{r}(h) K(n, j, z), \\
f_{r}(h) & =\mu_{r}(h) f(h) .
\end{aligned}
$$

Consider the Volterra equation

$$
x(n)=f_{r}(n)+\sum_{j=1}^{n} K_{r}(n, j, x(j)), \quad n \geq 0 .
$$

Due to Lemma 9 and condition (26), any solution of (46) satisfies (25) and therefore belongs to $\Omega_{r}$. But $K_{r}=K$ and $f_{r}=f$ on $\Omega_{r}$. This proves estimates (25) for a solution of (4). Estimates (24) follows from Lemma 7. This completes the proof of Theorem 6 .

\section{Volterra Difference Equations with Infinite Delay}

Consider the Volterra difference equation of the form

$$
x(n)=f(n)+\sum_{j=-\infty}^{n} K(n, j, x(j)), \quad n \geq 0,
$$

which can be regarded as a retarded equation whose delay is infinite. In general this problem requires that one give an "initial function" on $(-\infty, 0]$, in order to obtain a unique solution, after which the equation can be treated with the techniques of standard Volterra equations. The nonuniqueness of solutions of (47) is an intrinsic feature (e.g., see [25]). If $\varphi:(-\infty, 0] \rightarrow X$ is an initial function to (47), then we write (47) as

$$
\begin{aligned}
x(n)= & \left(f(n)+\sum_{j=-\infty}^{0} K(n, j, \varphi(j))\right) \\
& +\sum_{j=1}^{n} K(n, j, x(j)) \\
= & \tilde{f}(n)+\sum_{j=1}^{n} K(n, j, x(j)),
\end{aligned}
$$

so that the initial function $\varphi$ becomes part of the sequence $\{\tilde{f}(n)\}$. Under these conditions, we can apply Theorem 5 to (48), so that the next result is obtained.

Theorem 10. Assume that $\tilde{f} \in l_{p}\left(Z^{+}, X\right), 1 \leq p \leq \infty$, and condition (21) holds. Then the solution $x(n)=x(n, \varphi)$ of (48) belongs to $l_{p}\left(Z^{+}, X\right)$ and satisfies the inequalities

$$
\begin{aligned}
\|x\|_{p} & \leq m_{p}\left(A_{r}\right)\|\tilde{f}\|_{p}, \\
\|x\|_{\infty} & \leq\|\tilde{f}\|_{\infty}+\beta_{p}\left(A_{r}\right) m_{p}\left(A_{r}\right)\|\tilde{f}\|_{p} .
\end{aligned}
$$

Proof. Proceeding in a similar way to the proof of Theorem 6, with

$$
\tilde{f}(n)=f(n)+\sum_{j=-\infty}^{0} K(n, j, \varphi(j))
$$

instead of $f$, and choosing an appropriate initial function $\varphi$ such that $\widetilde{f} \in l_{p}\left(Z^{+}, X\right)$, the required result follows.

Remark 11. Consider

$$
\begin{aligned}
x(n) & =x(n, \varphi) \quad \forall n \geq 0, \\
x(j, \varphi) & =\varphi(j) \quad \text { for } j<0 .
\end{aligned}
$$

\section{Conclusions}

New conditions for the existence, uniqueness, and boundedness of solutions of infinite-dimensional nonlinear Volterra difference systems are derived. Unlike the classic method of stability analysis, we do not use the technique of the Lyapunov functions in the process of construction of the estimates for the solutions. The proofs are carried out using estimates for the norm of powers of quasi-nilpotent operators. That is, we interpreted the Volterra difference equations, with nonlinear kernels, as operator equations defined in the Banach spaces $l_{p}\left(Z^{+}, X\right)$. We want to point out that the results of this paper can be useful for discussions of $l_{p}\left(Z^{+}, X\right)$ stability of the zero solution of the homogeneous Volterra equation corresponding to (4).

In connection with the above investigations, some open problems arise. The richness of the spectral properties of operators acting on infinite-dimensional Hilbert spaces will need new stability formulations. Consequently, natural directions for future research is the generalization of our results to local exponential stabilizability of nonlinear Volterra difference equations or investigating the feedback stabilization of implicit nonlinear Volterra systems defined by operator Volterra equations.

\section{Competing Interests}

The author declares that there is no conflict of interests regarding the publication of this paper.

\section{Acknowledgments}

This research was supported by Dirección de Investigación under Grant NU06/16.

\section{References}

[1] A. D. Drozdov and V. B. Kolmanovskii, Stability in Viscoelasticity, vol. 38, Elsevier, Amsterdam, The Netherlands, 1994.

[2] N. Levinson, "A nonlinear Volterra equation arising in the theory of superfluidity," Journal of Mathematical Analysis and Applications, vol. 1, no. 1, pp. 1-11, 1960.

[3] V. B. Kolmanovskii and A. I. Matasov, "An approximate method for solving optimal control problem in hereditary systems," Doklady Mathematics, vol. 55, no. 3, pp. 475-478, 1997.

[4] P. Borne, V. Kolmanovskii, and L. Shaikhet, "Stabilization of inverted pendulum by control with delay," Dynamic Systems and Applications, vol. 9, no. 4, pp. 501-514, 2000.

[5] A. D. Myshkis, "General theory of Differential equations with delay," American Mathematical Society Translations, vol. 55, pp. $1-62,1951$. 
[6] N. V. Minh, "On the asymptotic behaviour of Volterra difference equations," Journal of Difference Equations and Applications, vol. 19, no. 8, pp. 1317-1330, 2013.

[7] N. V. Minh, "Asymptotic behavior of individual orbits of discrete systems," Proceedings of the American Mathematical Society, vol. 137, no. 9, pp. 3025-3035, 2009.

[8] N. V. Minh, "Asymptotic behavior of individual orbits of discrete systems," Proceedings of the American Mathematical Society, vol. 137, no. 9, pp. 3025-3035, 2009.

[9] Y. Nagabuchi, "Decomposition of phase space for linear Volterra difference equations in a Banach space," Funkcialaj Ekvacioj, vol. 49, no. 2, pp. 269-290, 2006.

[10] S. Murakami and Y. Nagabuchi, "Stability properties and asymptotic almost periodicity for linear Volterra difference equations in a Banach space," Japanese Journal of Mathematics, vol. 31, no. 2, pp. 193-223, 2005.

[11] C. González, A. Jiménez-Melado, and M. Lorente, "Existence and estimate of solutions of some nonlinear Volterra difference equations in Hilbert spaces," Journal of Mathematical Analysis and Applications, vol. 305, no. 1, pp. 63-71, 2005.

[12] Y. Song and C. T. Baker, "Linearized stability analysis of discrete Volterra equations," Journal of Mathematical Analysis and Applications, vol. 294, no. 1, pp. 310-333, 2004.

[13] Y. Muroya and E. Ishiwata, "Stability for a class of difference equations," Journal of Computational and Applied Mathematics, vol. 228 , no. 2, pp. 561-570, 2009.

[14] I. Győri and L. Horváth, "Asymptotic representation of the solutions of linear Volterra difference equations," Advances in Difference Equations, vol. 2008, Article ID 932831, 2008.

[15] V. B. Kolmanovskii, A. D. Myshkis, and J.-P. Richard, "Estimate of solutions for some Volterra difference equations," Nonlinear Analysis. Theory, Methods \& Applications. An International Multidisciplinary Journal. Series A: Theory and Methods, vol. 40, no. 1-8, pp. 345-363, 2000.

[16] Y. Song and C. T. H. Baker, "Perturbation theory for discrete Volterra equations," Journal of Difference Equations and Applications, vol. 9, no. 10, pp. 969-987, 2003.

[17] M. R. Crisci, V. B. Kolmanovskii, E. Russo, and A. Vecchio, "Stability of continuous and discrete Volterra integro-differential equations by Liapunov approach," Journal of Integral Equations and Applications, vol. 7, no. 4, pp. 393-411, 1995.

[18] S. N. Elaydi, An Introduction to Difference Equations, Undergraduate Texts in Mathematics, Springer, New York, NY, USA, 1996.

[19] M. Kwapisz, "On $l^{\mathrm{P}}$ solutions of discrete Volterra equations," Aequationes Mathematicae, vol. 43, no. 2-3, pp. 191-197, 1992.

[20] R. Medina, "Solvability of discrete Volterra equations in weighted spaces," Dynamic Systems and Applications, vol. 5, no. 3, pp. 407-421, 1996.

[21] R. Medina, "Stability results for nonlinear difference equations," Nonlinear Studies, vol. 6, no. 1, pp. 73-83, 1999.

[22] C. Corduneanu, Functional Equations with Causal Operators, vol. 16, Taylor and Francis, London, UK, 2002.

[23] M. I. Gil', Norm Estimations for Operator-Valued Functions and Applications, vol. 192 of Monographs and Textbooks in Pure and Applied Mathematics, Marcel Dekker, New York, NY, USA, 1995.

[24] N. Dunford and J. T. Schwarz, Linear Operators, Part I, Wiley Interscience, New York, NY, USA, 1966.

[25] P. Rejto and M. Taboada, "Unique solvability of nonlinear Volterra equations in weighted spaces," Journal of Mathematical Analysis and Applications, vol. 167, no. 2, pp. 368-381, 1992. 


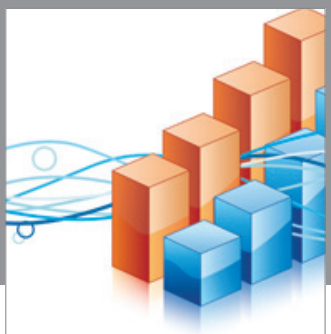

Advances in

Operations Research

vatem alat4

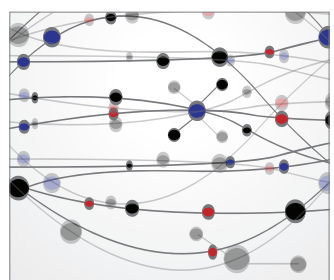

\section{The Scientific} World Journal
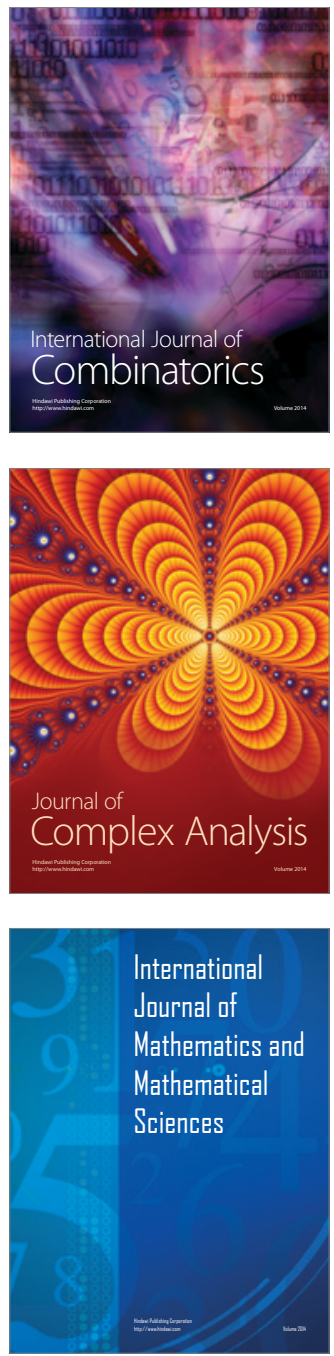
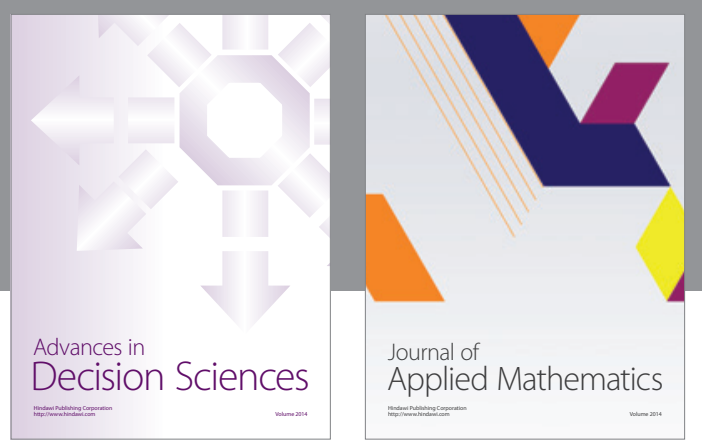

Algebra

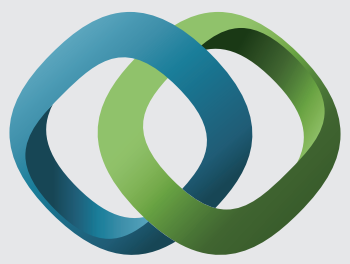

\section{Hindawi}

Submit your manuscripts at

http://www.hindawi.com
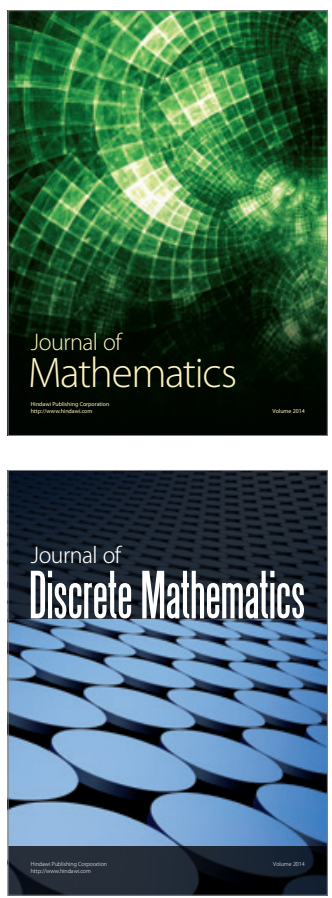

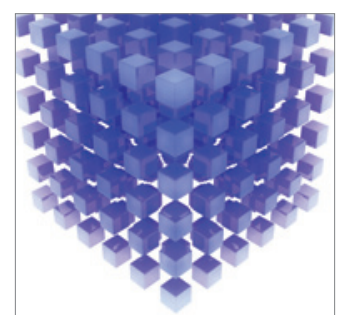

Mathematical Problems in Engineering
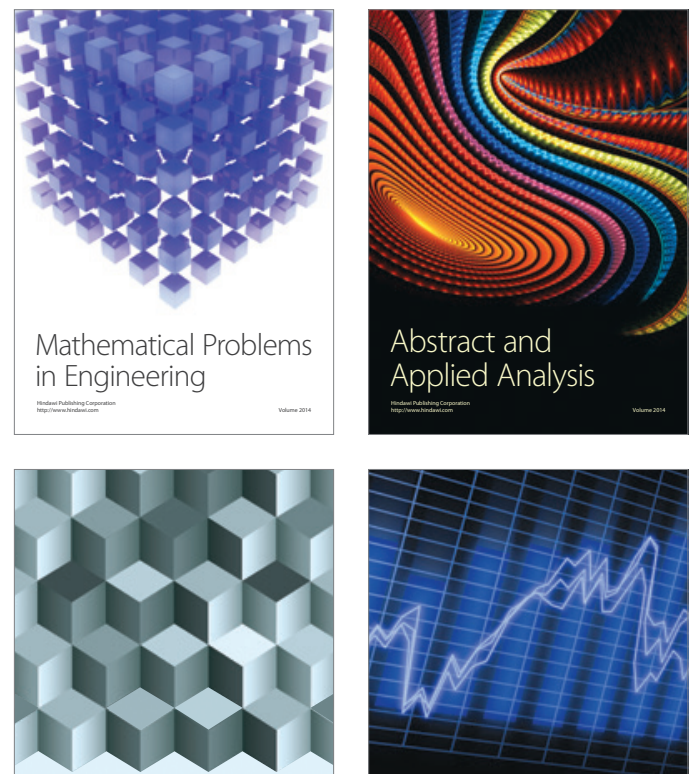

Journal of

Function Spaces

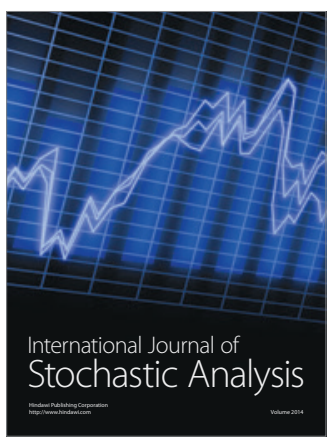

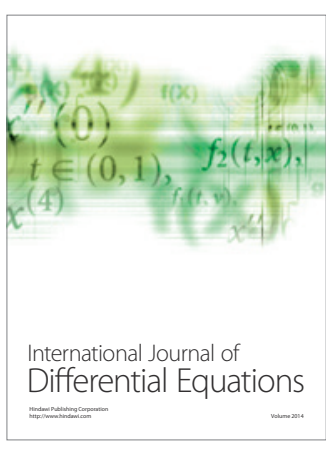
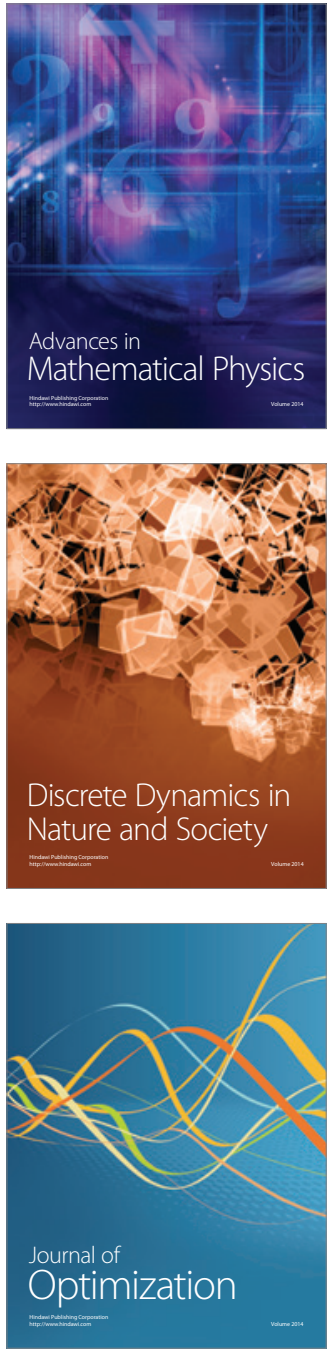